\title{
Decolorization of Azodyes by Chemically and Biosynthesized ZnO Nanoparticles
}

\section{Jasmine Swain}

Mount Carmel College https://orcid.org/0000-0002-5767-6170

Padmashree Kulkarni ( $\nabla$ padmashree.kulkarni@mccblr.edu.in )

Mount Carmel College

Suba Manuel

Mount Carmel College

\section{Research Article}

Keywords: Nanobioremediation, Azodye, Bacillus subtilis, ZnO nanoparticles, Decolorization

Posted Date: July 20th, 2021

DOI: https://doi.org/10.21203/rs.3.rs-722394/v1

License: (1) This work is licensed under a Creative Commons Attribution 4.0 International License. Read Full License 


\title{
Decolorization of Azodyes by Chemically and Biosynthesized ZnO Nanoparticles
}

\author{
Jasmine Swain $^{1}$, Padmashree Kulkarni ${ }^{1 *}$, Suba Manuel ${ }^{2}$ \\ ${ }^{1,2}$ Department of Life Science, Mount Carmel College, Autonomous, Bengaluru, India.
}

\begin{abstract}
Dye contaminants in industrial effluents contribute significantly to environmental pollution. Conventional waste water treatment methods are expensive and energy consuming. These limitations could be overcome by the use of nanobioremediation processes. The present work was an effort to study decolorization of azodyes by $\mathrm{ZnO}$ nanoparticles (NPs). Rubine GDB (R-GDB) and Congo Red (CR) were the azodyes selected for the study. The ZnO NPs were successfully synthesized by chemical and biological methods. Chemical synthesis of $\mathrm{ZnO}$ NPs was carried out by co-precipitation method; biological synthesis was done using bacteria Bacillus subtilis. The synthesized nanoparticles were characterized by UV-Vis Spectroscopy, SEM and XRD. The UV spectrophotometer showed peaks in the range of 300-400 nm. SEM pictures indicated the presence of NPs in the size of $100 \mathrm{~nm}-200 \mathrm{~nm}$. XRD results were analyzed on the basis of the peaks that were seen. EDX analysis showed the presence of $\mathrm{Zn}$ particles and oxygen particles majorly. Decolorization efficiency was evualated by calculating \% decolorization by Meyer's method. Chemically synthesized NPs showed maximum \% decolorization of the R-GDB (89.058 \%) and CR (88.888\%). The biosynthesized NPs showed the least decolorization (R-GDB -18\% and CR $20.357 \%$ ). However, moderate percentages of decolorization of both the azodyes were observed when a combination of the NPs was used (R-GDB -36.072\% and CR- $39.824 \%$ ). Nanoparticles showed good potential for the decolorization of the azo dyes. With further optimization of the parameters the present findings show that dye decolorization by chemically synthesized $\mathrm{ZnO}$ NPs could be used as a probable nanobioremediation approach to treat wastewaters.
\end{abstract}


Keywords: Nanobioremediation, Azodye, Bacillus subtilis, $\mathrm{ZnO}$ nanoparticles, Decolorization

\section{Declaration}

\section{Acknowledgments}

We gratefully acknowledge the CeNSE lab members (Indian Institute of Science, Bengaluru) for helping us with XRD and SEM facility. Thanks to Mr Pradeep, Mr Amit, Mr Abhishek, Ms Vanitha, Mr Arun Babu and Ms Suma for the guidance to conduct and analyse the XRD and SEM results.

\section{Code Availability}

Not applicable.

\section{Funding}

Not applicable.

\section{Author information}

\section{Affiliations}

- Department of Life Science, Mount Carmel College (Autonomous), Bengaluru, India Jasmine Swain- https://orcid.org/0000-0002-5767-6170

Dr. Padmashree Kulkarni- padmashree.kulkarni@mccblr.edu.in

Dr. Suba G A Manuel- https://www.researchgate.net/profile/Suba-Manuel

\section{Corresponding author}

Correspondence to

Dr. Padmashree Kulkarni 
padmashree.kulkarni@mccblr.edu.in

\section{Ethics declarations}

Ethics Approval

Not applicable.

\section{Consent to Participate}

Not applicable.

\section{Consent for Publication}

The participants gave their consent for publication.

\section{Conflict of Interest}

The authors declare no competing interests

\section{Introduction:}

Wastewater discharges polluting the environment is a matter of great concern and challenge. This issue is being faced throughout the world. India, along with other developing countries, faces a huge part of this worry as hardly any treatment is being carried out before the discharge. The cause of all of these issues is industries ${ }^{1,2,3}$. The disposal of wastewater generated from these industries is an alarming threat to nature. It accounts for $15-20 \%$ of the total wastewater in the country ${ }^{4}$. Azo dyes are a large group that are most commonly used in dyeing textile fibres i.e. cotton, wool, silk and synthetic fibres. They are the most common as they are affordable, easy to use and provide strong colours that are easily distinguished. There are approximately 2000 dyes available. Around 4-5\% azo dyes cleave to form aromatic amines 5 . 
Cancer and aromatic amines are found to be in close relation. Thus, azo dyes owe their carcinogenic and detrimental properties to the presence of aromatic amines and their water soluble nature. Therefore, it is easy for the body to absorb and cause harm ${ }^{5}$. Rubine-GDB (RGDB) is an azo dye that is used to impart red colour to fabrics. It is also used in paints, printing inks, textile printing and plastics. It is carcinogenic in nature. Congo Red (CR) is an azo dye that is water soluble in nature. In earlier times, it was used to impart a lighter shade of red colour to cotton which then became more popular as a staining agent in histology and as an acid-base indicator. It is also carcinogenic in nature with greater solubility in organic solvents. The most effective and substantially efficient methods till date have been the biological techniques ${ }^{6}$. Consistent research has been taking place with respect to developing newer and better techniques for the degradation of dyes.

Nanotechnology is currently a booming industry and diversified kinds of studies are being carried out at this point. One such aspect is the use of nanoparticles (NPs) for the treatment of wastewater released from industries. The synthesis of NPs can be carried out by various physical, chemical and biological methods. The biosynthesized NPs have drawn enormous focus due to their non-toxic and eco-friendly nature. Consequently, bionanotechnology is now regarded as the epitome of producing eco-friendly and cost effective nanoparticles for the treatment of wastewater and dye degradation ${ }^{7}$. NPs have structural dimension of less than $100 \mathrm{~nm}$ which makes them comparable to sub-cellular structures thereby enabling them to get incorporated into biological systems ${ }^{8}$. Zinc oxide NPs have a large surface area and high catalytic activity. These nanoparticles have different physical and chemical properties in accordance with the source of their synthesis. Recently, these ZnO NPs have gained much acclamation for dye degradation procedures ${ }^{9,10,11 .}$

Biological synthesis of NPs using bacteria has been of special interest as it involves easy handling procedures, eco-friendly disposal methods and very easy for downstream 
processing. Recently, ecofriendly synthesis of metal NPs using various strains of bacteria such as Bacillus species and their use in dye decolorization and degradation has been reported $^{12}$. Present investigation was an effort to study the efficiency of azo dye decolorization of R-GDB and CR by ZnO NPs synthesized following chemical and biological routes.

\section{Materials and Methods}

Chemical synthesis was carried out by co-precipitation method and biological synthesis was done using Bacillus subtilis.

Synthesis of ZnO NPs by chemical route: Zinc nitrate hexahydrate (ZnNO3.6H2O;0.1M) and Sodium hydroxide $(\mathrm{NaOH} ; 0.8 \mathrm{M})$ solutions were prepared in distilled water. After complete dissolution of zinc nitrate, $\mathrm{NaOH}$ was added drop-wise with continuous stirring for 45 min. A white colloid was formed which was allowed to settle overnight. Supernatant was separated carefully and the remaining solution was centrifuged to obtain the precipitate. Precipitate was collected, washed with de-ionized water and ethanol to remove the byproducts. It was later subjected for hot air oven drying at about $60^{\circ} \mathrm{C}$ for 2-3 days ${ }^{13}$. Absorption Spectrum was checked for the presence of NPs. The powdered NPs were allowed for SEM and XRD measurements.

Synthesis of ZnO NPs by biological route: Zinc acetate dihydrate $(\mathrm{Zn}(\mathrm{CH} 3 \mathrm{COO}) 2 \cdot 2 \mathrm{H} 2 \mathrm{O}$; $0.001 \mathrm{M}$ ) was added into $B$. subtilis inoculum prepared in nutrient broth and was incubated at $37^{\circ} \mathrm{C}$ overnight. The culture was centrifuged at $5000 \mathrm{rpm}$ for $15 \mathrm{~min}$. Pellet was collected, washed and was dried completely in a hot air oven at $60^{\circ} \mathrm{C}$ until a fine powder was obtained. The size and morphology of the powder was studied by scanning electron microscope (SEM) and $\mathrm{x}$-ray diffraction $(\mathrm{XRD})$ was recorded ${ }^{14}$. 


\section{Characterization of nanoparticles}

UV-Vis Spectroscopy: $\mathrm{ZnO}$ nanoparticles obtained by the chemical synthesis, biological synthesis were analyzed in the UV-Visible spectrophotometer (Shimadzu, UV-1700 Pharma Spec). It does so by confirming the range at which the peak would be formed. The setup is made by preparing a suspension of the NPs in milli-q water. Small aliquots of the same are then taken in the cuvette and the analysis is carried out.

Scanning Electron Microscope (SEM) analysis: The surface structure, shape and size of the NPs were analyzed by SEM studies ${ }^{15}$. This was performed using Zeiss, Ultra 55 Field Emission Scanning Electron Microscope at CeNSE laboratory, Indian Institute of Science (IISc), Bengaluru. SEM studies confirmed the presence of B. subitilis bound $\mathrm{ZnO}$ nanoparticles in the range of $200-300 \mathrm{~nm}$.

XRD analysis: Dried powdered NPs were subjected for the XRD analysis. The crystallite structure analysis was done using Rigaku x-ray diffractometer at CeNSE laboratory, IISc, Bengaluru. The crystallite size was determined with the use of Scherrer's equation,

$$
\mathrm{D}=\frac{K \lambda}{\beta \cos \theta}
$$

where is $D$ is average crystallite size, $K$ is Scherrer constant, $\theta$ is Bragg angle, $\beta$ is Line broadening in radians and $\lambda$ is X-Ray wavelength ${ }^{16}$.

Energy Dispersive X-ray Spectroscopy (EDX): The elemental composition, chemical purity and stoichiometry of the synthesized $\mathrm{ZnO}$ nanoparticles were estimated by the EDX analysis $^{15}$. It is performed by using Zeiss, Ultra 55 Field Emission Scanning Electron Microscope at CeNSE laboratory, IISc., Bengaluru. 
Decolorization of azodyes by NPs: Stock solutions of the azodyes at a concentration of $1 \mathrm{mgmL}^{-1}$ were prepared in milli q water. Decolorization studies were carried out in three sets in a closed batch culture system at room temperature $\left(28 \pm 2^{\circ} \mathrm{C}\right)$. NPs were added to the dye solutions taken in glass screw capped tubes at a concentration of $250 \mathrm{ppm}$ with continuous stirring at $75 \mathrm{rpm}$ on a rotary shaker. In the first set ZnO NPs synthesized chemically were used. In the second set NPs synthesized biologically by B. subtilis were used. In the third set both chemically and biologically synthesized NPs were used. Samples were withdrawn every 24 hours, centrifuged at $3000 \mathrm{rpm}$ for 10 minutes. Clear supernatant was pipetted and absorbance was read at $\lambda_{\max }$ of $580 \mathrm{~nm}$. Dye decolorization percentage was calculated by the following formula ${ }^{17}$ :

$$
\text { Decolorization }(\%)=\frac{\text { Initial OD }- \text { Final OD }}{\text { Initial OD }} \text { X100 }
$$

\section{Results and Discussion}

NPs were successfully synthesized following chemical and biological routes. During biological synthesis reduction of zinc ions into zinc NPs occurs on exposure to bacterial cell extracts as a result a colour change occurred. Generally the metal NPs have free electrons, which help in the formation of the Surface Plasmon Resonance absorption band. It happens due to the united vibration of the electrons of metal nanoparticles in resonance with light wave $^{18}$.

\section{Characterization of $\mathrm{ZnO}$ NPs}

The synthesized NPs were characterized through the instrumental analysis such as UVVisible spectroscopy, X-Ray Diffraction (XRD), Scanning Electron Microscope (SEM), Energy Dispersive X-ray analysis (EDX). 
UV absorption analysis of ZnO NPs: Using UV-Vis spectroscopy the absorption spectra of $\mathrm{ZnO}$ NPs was obtained. The maximum absorption $\left(\lambda_{\max }\right)$ was obtained in the range of $380 \mathrm{~nm}$ to $390 \mathrm{~nm}$ for chemical $\mathrm{ZnO}$ NPs and $370 \mathrm{~nm}$ to $380 \mathrm{~nm}$ for biosynthesized NPs. The results obtained in the present study show similarities with the findings of previous literature where they reported absorption maxima in a range of 320-390 $\mathrm{nm}$ which is a characteristic band for the pure $\mathrm{ZnO}^{19}$.

X-ray diffraction (XRD) analysis: XRD patterns for the NPs is indicated in Figure 4 and 5. The crystallite size of chemically synthesized NPs determined by Scherrer's equation was $24.419 \mathrm{~nm}$. Graphs obtained were compared with the standard graph. A sharp peak was obtained at 101 (Figure 1). This confirms the sample to be ZnO NPs in polycrystalline form. However, biologically synthesized NPs were amorphous in nature and no crystals were formed. Hence the size of crystallite size could not be estimated since a clear graph was not obtained. This could be due to the presence of impurities in the sample (Figure 2). Broadening line of the diffraction peaks indicates that the synthesized particles are in $\mathrm{nm}$ range $^{20}$. Peaks obtained other than the characteristic peaks are also due to impurities.

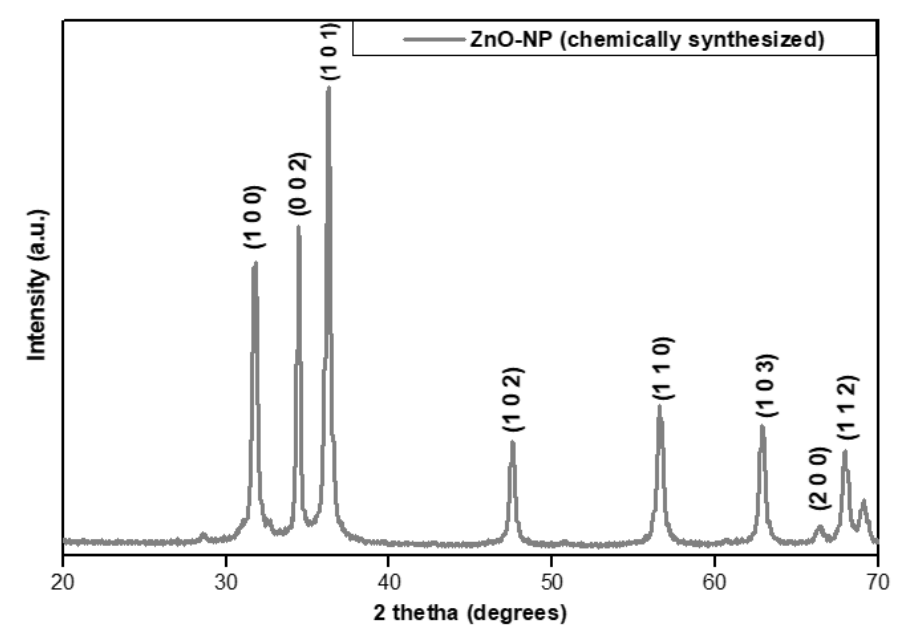

Fig.1: XRD graph of chemically synthesized ZnO-NPs 


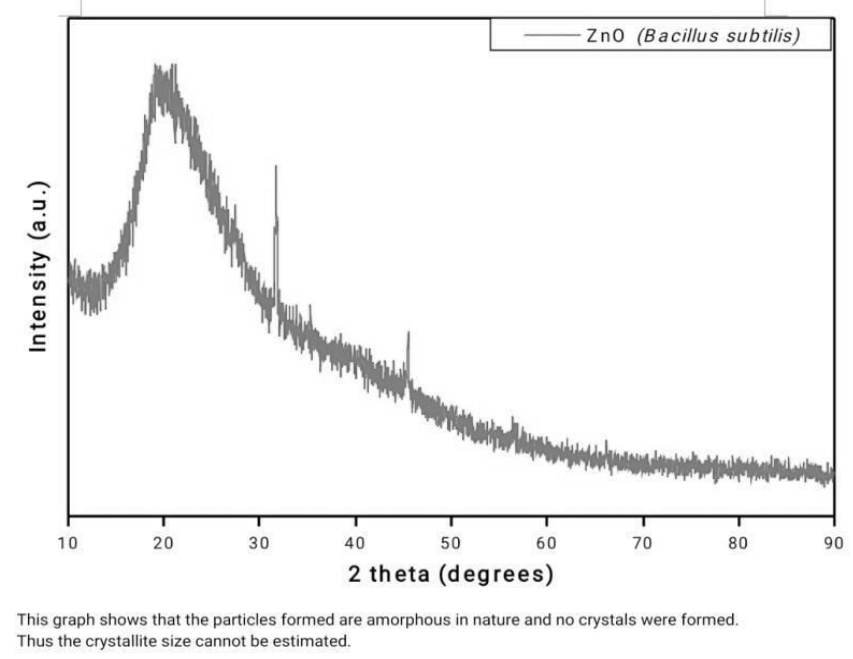

Fig. 2: XRD graph of ZnO-NPs synthesized using Bacillus subtilis strain PBA

\section{SEM analysis of $\mathrm{ZnO}$ particles:}

The SEM images of chemically synthesized ZnO NPs showed spherical non-agglomerated, uniform shaped and evenly distributed nano flakes as seen in the image (Figure 3). The average diameter of the structures ranged from $100 \mathrm{~nm}-200 \mathrm{~nm}$. Images are similar to previous literature having ${ }^{21}$.

SEM images of biosynthesized NPs showed nanorod shape embeded nanopaticle structures with hints of agglomeration in the bacterial biomass. The average diameters of the stuctures were in the range of $100 \mathrm{~nm}-200 \mathrm{~nm}$. The structures are seen to be distributed evenly and are of similar shapes. These images can be correlated with the previous literature ${ }^{22}$. Synthesis of NPs using bacteria has been studied by several researchers but the exact mechanism is till not clear, however cells of bacteria play role in the formation of $\mathrm{NPs}^{23}$. 

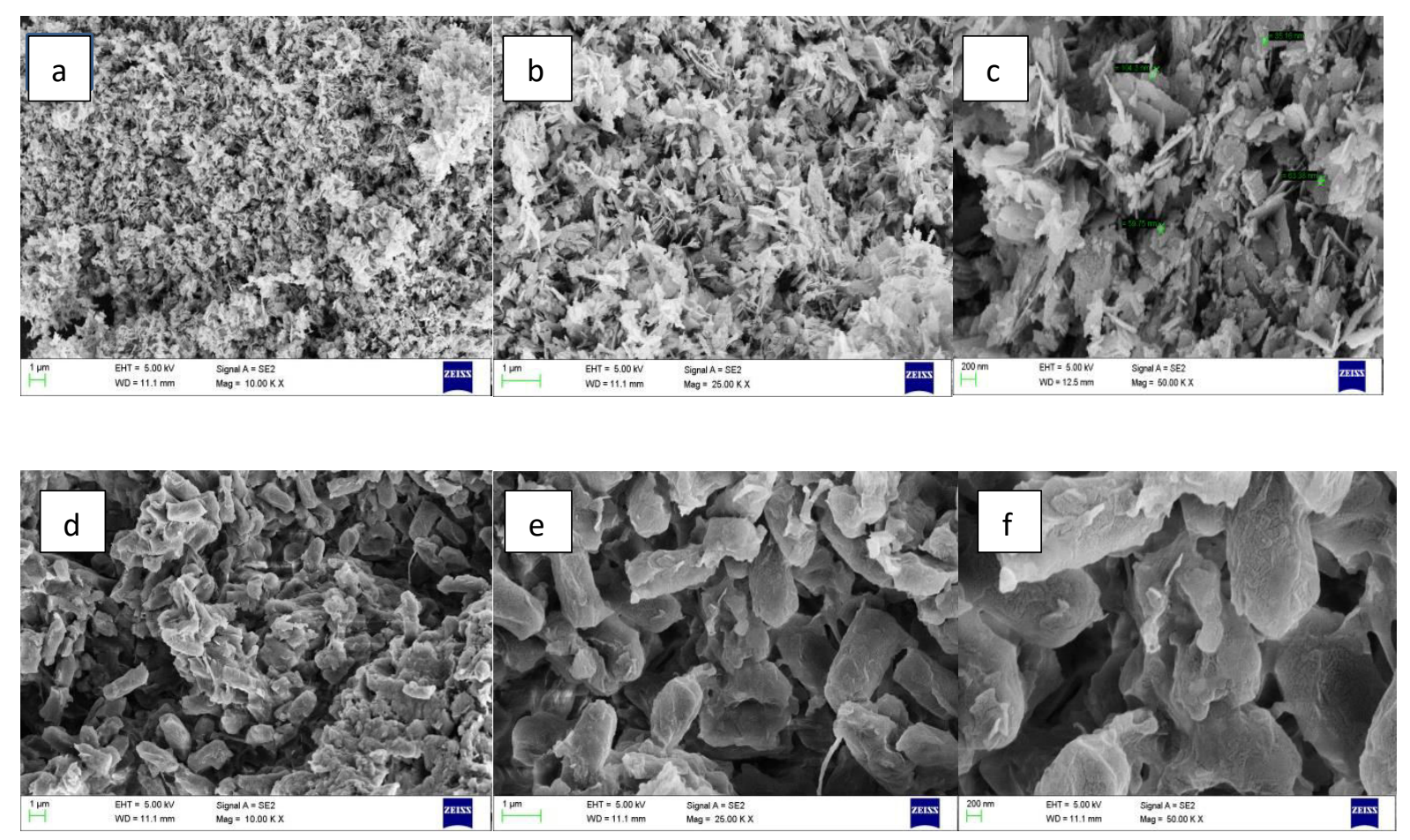

Fig. 3 : SEM image of ZnO NPs (a) Chemically synthesized,10.00 KX, (b) Chemically synthesized, $25.00 \mathrm{KX}$, (c) Chemically synthesized, $50.00 \mathrm{KX}$, (d) Biosynthesized ,10.00 KX, (e) Biosynthesized , $25.00 \mathrm{KX}$, (f) Biosynthesized, $50.00 \mathrm{KX}$.

\section{Energy Dispersive X-ray analysis (EDX)}

From the results obtained it can be seen that the sample is purely made of $\mathrm{ZnO}$, major amounts of $\mathrm{Zn}$ (Zinc) and $\mathrm{O}$ (Oxygen) to be present in the sample. Carbon present in the sample is due to the carbon tape which was used as sample holder. Hence it can be said that the purification has been carried out well. Since the weight $\%$ is 65.95 , the sample had fairly 65-66\% of $\mathrm{Zn}$ in it (Table 1). Characteristic peaks of $\mathrm{Zn}$ and $\mathrm{O}$ confirm the presence of $\mathrm{ZnO}$ NPs with no impurities ${ }^{21}$.

In Biological sample zinc and oxygen signals indicated that the synthesized nanoflakes have a major amount of $\mathrm{Zn}$ (Zinc) and $\mathrm{O}$ (Oxygen). Presence of carbon can be due to carbon tape used during analysis. Trace amounts of impurities were also detected in the analysis which 
indicate that the samples were impure. Since the weight $\%$ is 65.97 , the sample had fairly 65 $66 \%$ of $\mathrm{Zn}$ in it (Table 1). The impurities were detected because biological molecules combine with the NPs in the bacteria ${ }^{24}$.

Degradation of dye using NPs: Azodye decolorization was observed in all the three sets of NPs. Decolorization percentages of R-GDB by the NPs at 48 hours, 72 hours and 96 hours of incubation is indicated in Figure 4.

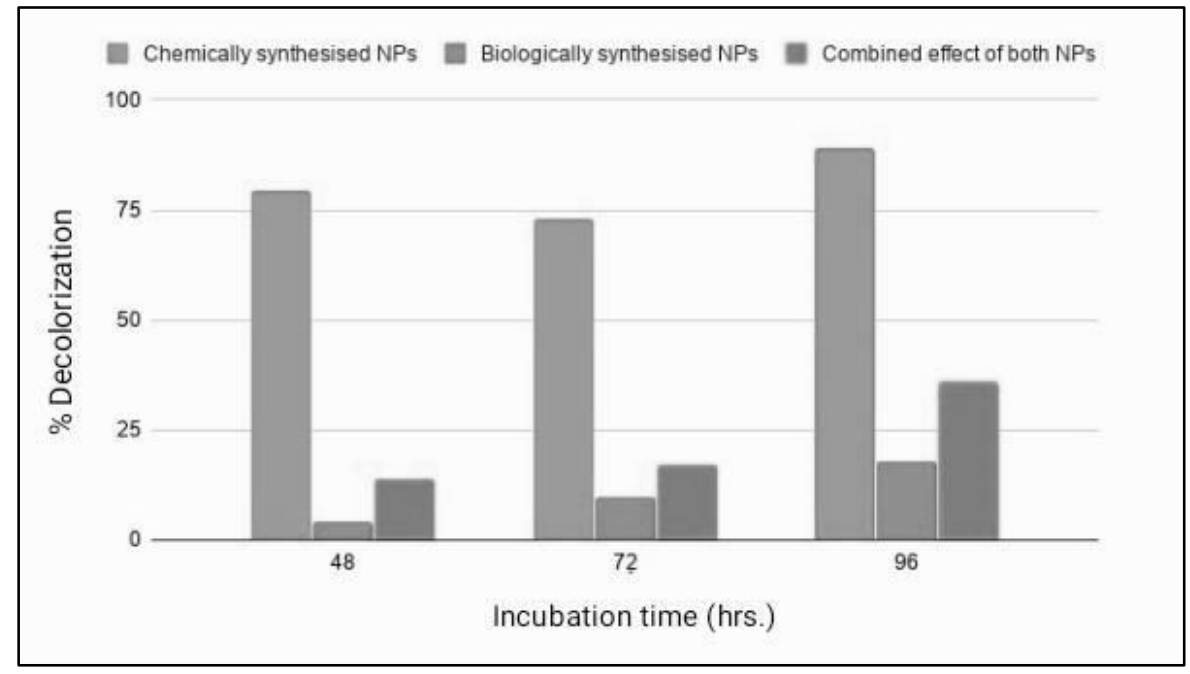

Fig. 4: Decolorization (\%) of Rubine GDB with chemically and biologically synthesized NPs at different times of incubation (hours)

For Rubine dye, as is evident from Table 2 and Figure 4 the maximum decolorization was observed at 96 hours of incubation. The was seen with Chemically synthesized NPs (89.058 $\%)$ showed the maximum \% decolorization followed by combination of both NPs (18\%) and then the biosynthesized NPs $(36.072 \%)$. 


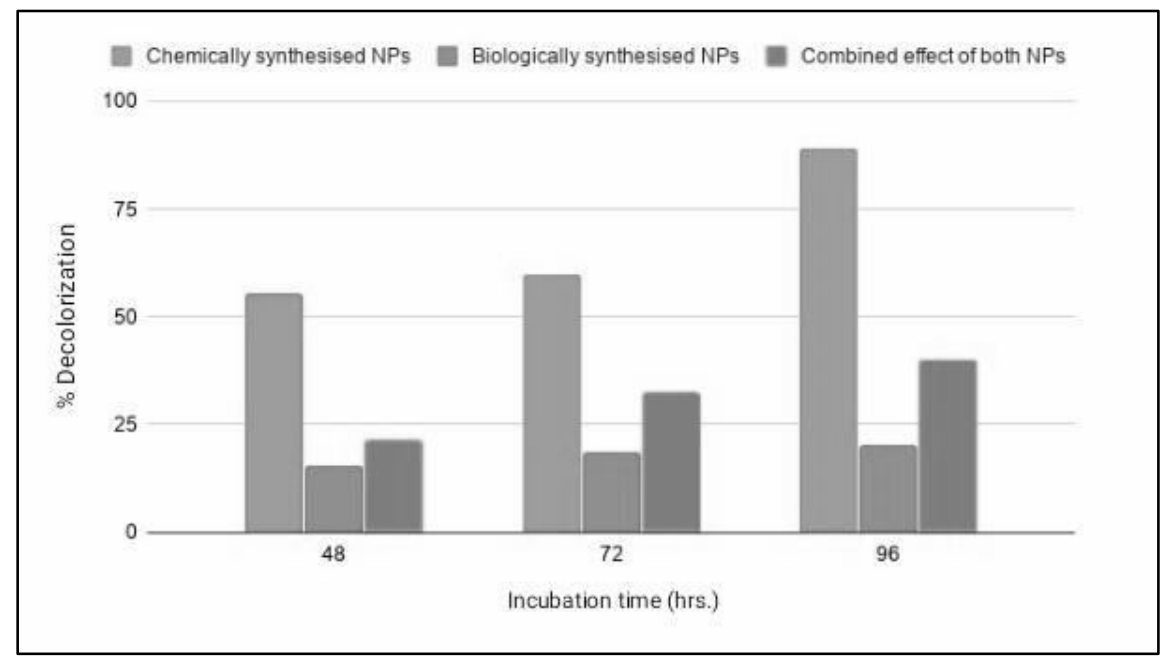

Fig. 5: Decolorization (\%) of Congo Red by chemically, biologically synthesized NPs at different times of incubation (hours)

Decolorization of CR by NPs: For CR dye, as is evident from Table 3 and Figure 5, the maximum decolorization was obtained at 96 hours of incubation. Chemically synthesized NPs showed maximum decolorization $(88.88 \%)$ followed by combination of both NPs $(39.82$ $\%)$ and then biosynthesized NPs (20.35\%).

Present findings of decolorization studies of azodye by NPs showed that chemically synthesized ZnO NPs could decolorize azodyes and can be recovered by simple methods ${ }^{25}$. Percent decolorization of the dye increased with an increase in the treatment duration. With the same concentration of NPs taken, \% decolorization increases with the treatment time. also more the number of NPs, the more is it likely for the catalyst (NPs) to attack the dye's chromopheric system ${ }^{26}$. Biologically synthesized NPs showed the least dye decolorization. The microbe mediated $\mathrm{ZnO}$ NPs are nontoxic, eco-friendly and biocompatible and are used as an alternative for chemical and physical practices ${ }^{27} . \mathrm{ZnO}$ is known to have antimicrobial properties hence this property of $\mathrm{ZnO}$ can inhibit the growth of Bacillus subtilis ${ }^{28}$. ZnO NPs have anti-bacterial properties and they tend to disrupt the cell membranes of bacteria ${ }^{29}$. Hence this might be the reason that both the dyes were not efficiently decolorized. 


\section{Conclusion}

Nanoparticles were successfully synthesized by chemically and biologically. Chemically syntheized NPs showed high percent decolorization of dyes-Rubine GDB (89.058 \%) and Congo Red $(88.888 \%)$. Hence they show great potential to be used for dye contaminated wastewater treatment. Biologically synthesized NPs showed least dye decolorization. A combination of both biologically and chemically synthesized NPs used in the treatment of dye samples also did not show satisfactory percentage of dye decolorization. Further research studies are required to study the mechanisms that are involved in biological synthesis of $\mathrm{ZnO}$ NPs using Bacillus subtilis. Present study was a venture to study azodye degradation by NPs as it is simple, cost-effective method.

\section{References:}

1. Govindarajalu, K., \& Govindarajalu, K. (2003, December). Industrial effluent and health status: A case study of Noyyal river basin. In Proceedings of the third international conference on environment and health, Chennai, India (Vol. 15, No. 17, pp. 150-157).

2. Appasamy, P. P., \& Nelliyat, P. (2007). Compensating the loss of ecosystem services due to pollution in Noyyal River basin, Tamil Nadu. Madras School of Economics.

3. Sivakumar, K. K., Balamurugan, C., Ramakrishnan, D., \& Bhai, L. H. (2011). Assessment studies on wastewater pollution by textile dyeing and bleaching industries at Karur, Tamil Nadu. Rasayan Journal of Chemistry, 4(2), 264-269.

4. Gopal, B. (1999). Natural and constructed wetlands for wastewater treatement: potentials and problems. Water science and technology, 40(3), 27-35.

5. Nijkamp, M. M., Maslankiewics, L., Delmaar, J. E., \& Muller, J. J. A. (2015). Hazardous substances in textile products.

6. Sarkar, S., Banerjee, A., Halder, U., Biswas, R., \& Bandopadhyay, R. (2017). Degradation of synthetic azo dyes of textile industry: a sustainable approach using microbial enzymes. Water Conservation Science and Engineering, 2(4), 121-131. 
7. Nomoto, K. I., Tominaga, N., Umeda, H., Kobayashi, C., \& Maeda, K. (2006). Nucleosynthesis yields of core-collapse supernovae and hypernovae, and galactic chemical evolution. Nuclear Physics A, 777, 424-458.

8. Orgeig, S., Morrison, J. L., Sullivan, L. C., \& Daniels, C. B. (2014). The development of the pulmonary surfactant system. In The Lung (pp. 183-209). Academic Press.

9. Kuo, C. L., Kuo, T. J., \& Huang, M. H. (2005). Hydrothermal synthesis of ZnO microspheres and hexagonal microrods with sheetlike and platelike nanostructures. The Journal of Physical Chemistry B, 109(43), 20115-20121.

10. El-Rafie, H. M., El-Rafie, M., \& Zahran, M. K. (2013). Green synthesis of silver nanoparticles using polysaccharides extracted from marine macro algae. Carbohydrate polymers, 96(2), 403-410.

11. Wei, M., Casey Boutwell, R., Faleev, N., Osinsky, A., \& Schoenfeld, W. V. (2013). Growth of high quality $\mathrm{ZnO}$ thin films with a homonucleation on sapphire. Journal of Vacuum Science \& Technology B, Nanotechnology and Microelectronics: Materials, Processing, Measurement, and Phenomena, 31(4), 041206.

12. Velusamy, P., Kumar, G. V., Jeyanthi, V., Das, J., \& Pachaiappan, R. (2016). Bio-inspired green nanoparticles: synthesis, mechanism, and antibacterial application. Toxicological research, 32(2), 95102.

13. Talam, S., Karumuri, S. R., \& Gunnam, N. (2012). Synthesis, characterization, and spectroscopic properties of ZnO nanoparticles. International Scholarly Research Notices, 2012. 2

14. Willner, I., Basnar, B., \& Willner, B. (2007). Nanoparticle-enzyme hybrid systems for nanobiotechnology. The FEBS journal, 274(2), 302-309.

15. Chaudhuri, S. K., \& Malodia, L. (2017). Biosynthesis of zinc oxide nanoparticles using leaf extract of Calotropis gigantea: characterization and its evaluation on tree seedling growth in nursery stage. Applied Nanoscience, 7(8), 501-512.

16. Becheri, A., Dürr, M., Nostro, P. L., \& Baglioni, P. (2008). Synthesis and characterization of zinc oxide nanoparticles: application to textiles as UV-absorbers. Journal of Nanoparticle Research, 10(4), 679-689

17. Nekouei, S., \& Nekouei, F. (2018). Comparative procedure of photodegradation of methylene blue using $\mathrm{N}$ doped activated carbon loaded with hollow 3D flower like $\mathrm{ZnS}$ in two synergic phases of adsorption and catalytic. Journal of Photochemistry and Photobiology A: Chemistry, 364, 262-273. 
18. Haes, A. J., \& Van Duyne, R. P. (2002). A nanoscale optical biosensor: sensitivity and selectivity of an approach based on the localized surface plasmon resonance spectroscopy of triangular silver nanoparticles. Journal of the American Chemical Society, 124(35), 10596-10604.

19. Jamdagni, P., Khatri, P., \& Rana, J. S. (2018). Green synthesis of zinc oxide nanoparticles using flower extract of Nyctanthes arbor-tristis and their antifungal activity. Journal of King Saud UniversityScience, 30(2), 168-175.

20. Selvarajan, E., \& Mohanasrinivasan, V. (2013). Biosynthesis and characterization of ZnO nanoparticles using Lactobacillus plantarum VITES07. Materials Letters, 112, 180-182.

21. Samanta, P. K., \& Saha, A. (2015). Wet chemical synthesis of ZnO nanoflakes and photoluminescence. Optik, 126(23), 3786-3788.

22. Bhakat, C., \& Singh, P. P. (2012). Zinc oxide nanorods: synthesis and its applications in solar cell. Int J Mod Eng Res, 2, 2452-2454.

23. Mahdi, Z. S., Talebnia Roshan, F., Nikzad, M., \& Ezoji, H. (2020). Biosynthesis of zinc oxide nanoparticles using bacteria: a study on the characterization and application for electrochemical determination of bisphenol A. Inorganic and Nano-Metal Chemistry, 1-9.

24. Arshad, A. (2017). Bacterial synthesis and applications of nanoparticles. Nanoscience and Nanotechnology, 11(2), 119.

25. Saleh, S. M. (2019). ZnO nanospheres based simple hydrothermal route for photocatalytic degradation of azo dye. Spectrochimica Acta Part A: Molecular and Biomolecular Spectroscopy, 211, 141-147.

26. Kale, R. D., \& Kane, P. B. (2017). Colour removal using nanoparticles. Textiles and Clothing Sustainability, 2(1), 1-7.

27. Yusof, H. M., Mohamad, R., \& Zaidan, U. H. (2019). Microbial synthesis of zinc oxide nanoparticles and their potential application as an antimicrobial agent and a feed supplement in animal industry: a review. Journal of animal science and biotechnology, 10(1), 1-22.

28. Padmavathy, N., \& Vijayaraghavan, R. (2008). Enhanced bioactivity of ZnO nanoparticles —an antimicrobial study. Science and technology of advanced materials.

29. Xie, Y., He, Y., Irwin, P. L., Jin, T., \& Shi, X. (2011). Antibacterial activity and mechanism of action of zinc oxide nanoparticles against Campylobacter jejuni. Applied and environmental microbiology, 77(7), 2325-2331 
Table 1: EDX analysis of chemically and biosynthesized ZnO NPs

\begin{tabular}{|c|c|c|c|}
\hline \multicolumn{2}{|c|}{ ZnO NPs } & $\begin{array}{c}\text { Chemical } \\
\text { synthesized }\end{array}$ & Biosynthesized \\
\hline & $\mathrm{Zn}$ & 65.95 & 65.97 \\
\cline { 2 - 4 } & $\mathrm{O}$ & 20.82 & 20.66 \\
\cline { 2 - 4 } & Other Impurities & 13.22 & 13.37 \\
\cline { 2 - 4 } Weight & Total & 100 & 100 \\
\hline \multirow{3}{*}{$\begin{array}{c}\text { Atomic } \\
\text { \% }\end{array}$} & $\mathrm{Zn}$ & 34.96 & 35.01 \\
\cline { 2 - 4 } & O & 45.10 & 44.81 \\
\cline { 2 - 4 } & Other Impurities & 19.93 & 20.01 \\
\hline
\end{tabular}

Table2: Decolorization (\%) of Rubine GDB with chemically, biosynthesized NPs and with the combination of both under different incubation time.

\begin{tabular}{|c|c|c|c|}
\hline $\begin{array}{c}\text { Incubation period } \\
\text { (hours.) }\end{array}$ & \multicolumn{3}{|c|}{ \% Decolorization $\left(\mathbf{2 5 0} \mathbf{\mu g m l}^{-1}\right.$ of NPs) } \\
\cline { 2 - 4 } & $\begin{array}{c}\text { Chemically synthesized } \\
\text { NPs }\end{array}$ & $\begin{array}{c}\text { Biosynthesized } \\
\text { NPs }\end{array}$ & $\begin{array}{c}\text { Combined effect of } \\
\text { NPs (Chemically } \\
\text { synthesized NPs + } \\
\text { Biosynthesized NPs) }\end{array}$ \\
\hline 48 & 55.537 & 15.357 & 21.428 \\
\hline 72 & 59.851 & 18.851 & 32.526 \\
\hline 96 & 88.888 & & 39.824 \\
\hline & & 20.357 & \\
\hline
\end{tabular}


Table 3: Decolorization (\%) of Congo Red with chemically, biosynthesized NPs and with the combination of both under different incubation time.

\begin{tabular}{|c|c|c|c|}
\hline \multirow{2}{*}{$\begin{array}{c}\text { Incubation period } \\
\text { (hours.) }\end{array}$} & \multicolumn{3}{|c|}{$\%$ Decolorization $\left(\mathbf{2 5 0} \mathbf{\mu g m l}^{-\mathbf{1}}\right.$ of NPs) } \\
\cline { 2 - 4 } & $\begin{array}{c}\text { Chemically synthesized } \\
\text { NPs }\end{array}$ & $\begin{array}{c}\text { Biosynthesized } \\
\text { NPs }\end{array}$ & $\begin{array}{c}\text { Combined effect of } \\
\text { NPs (Chemically } \\
\text { synthesized NPs + } \\
\text { Biosynthesized NPs) }\end{array}$ \\
\hline 48 & 55.537 & 15.357 & 21.428 \\
\hline 72 & 59.851 & 18.851 & 32.526 \\
\hline 96 & 88.888 & 20.357 & 39.824 \\
\hline
\end{tabular}

